\title{
Developing active personal learning environments on smart mobile devices
}

Conference or Workshop Item

Accepted Version

Whalley, B., France, D., Park, J., Mauchline, A. and Welsh, K. (2019) Developing active personal learning environments on smart mobile devices. In: Future Technologies Conference (FTC) 2019, 24-25 Oct 2019, San Franscisco, pp. 871-889. Available at http://centaur.reading.ac.uk/86350/

It is advisable to refer to the publisher's version if you intend to cite from the work. See Guidance on citing.

Published version at: https://link.springer.com/chapter/10.1007\%2F978-3-030-32523-7_64

All outputs in CentAUR are protected by Intellectual Property Rights law, including copyright law. Copyright and IPR is retained by the creators or other copyright holders. Terms and conditions for use of this material are defined in the End User Agreement. 


\section{CentAUR}

Central Archive at the University of Reading

Reading's research outputs online 


\title{
Developing active personal learning environments on smart mobile devices
}

\author{
Brian Whalley ${ }^{1}$, Derek France ${ }^{2}$, Julian Park ${ }^{3}$, Alice Mauchline ${ }^{4}$ and Katharine Welsh ${ }^{5}$ \\ ${ }^{1}$ University of Sheffield, Sheffield, S10 2TN, UK \\ b.whalley@sheffield.ac.uk \\ ${ }^{2}$ University of Chester, CH1 4BJ, UK \\ ${ }^{3}$ University of Reading, RG6 6HA, UK \\ ${ }^{4}$ University of Reading, RG6 6HA, UK \\ ${ }^{5}$ University of Chester, CH1 4BJ, UK
}

\begin{abstract}
Tablets' and other 'smart' devices (such as iPads and iPhones) have established themselves as a significant part of mobile technologies used in mobile (m-)learning. Smart devices such as iPads and the Apple Watch not only provide many apps that can be used for a variety of educational purposes; they also allow communication between students and tutors and with the world at large via social media. We argue that 'smart' mobile devices enable personalized learning by adjusting to the educational needs of individuals. We refer to Salmon's quadrat diagram to suggest where using mobile technologies should be of benefit to revising our views of pedagogy, making it much more responsive to students' needs in education as well as the world in general. Smart mobile devices now contain computing power to allow voice and face recognition, augmented reality and machine learning to make them intelligent enough to act as tutors for individual students and adjust and respond accordingly. To take advantage of these facilities on mobile devices, pedagogy must change from an institution-centred to a student-tutor-device focus. This is best done via 'active learning' and incorporating cognitive awareness into an educational operating system that can develop with the owner.
\end{abstract}

Keywords: Personal learning environments, Smart devices, Quality enhancement, supercomplexity.

\section{Educational Scenarios}

\subsection{Some Quotidian Scenes}

Consider the following:

A server in a restaurant takes a break to study for a class

A grandmother sends a letter to her grandson 
A farmer in Africa looks for a market for her product

A visitor to a foreign country grapples with translating a conversation

A mother with visual impairment reads a book to her child

A tutor explains a difficult concept to her dyslexic student

A student takes notes from a teacher's class talk.

These are all situations that happen every day somewhere in the world and may well have been occurring for the last hundred years (and more). They are all, in one sense or another, 'educational' and also 'one-to-one' if not 'face-to-face'. Talking and listening, writing and reading; the fundamental ways of information-knowledge transfer. Technology has transformed these in the lifetime of a centenarian; from telegraph to telephone, radio and TV, cinema through to e-mail the internet and the smart devices now familiar around the world. Smartphones have become ubiquitous in todays' information transfer system.

An exception to the list might be the student taking notes from a teacher. The UK's Open University (OU) was set up in 1969 and was an early example of providing flexible distance learning. It originally used short formal lectures or demonstrations delivered via TV and supplemented by local tutors, summer schools and course textbooks. For the most part, OU course materials could be studied informally, by the restaurant server for example. Today, an institutional virtual learning environment (VLE) or learning management system (LMS) is the main way in which higher education (HE) is most frequently 'delivered' to students as E-learning perhaps coupled to traditional lectures. This paper examines recent developments in mobile technologies in HE institutions (HEIs). In particular, we examine how smart devices can promote active and involved learning by students to supplement or replace these traditional forms of educational delivery. We suggest pedagogically sound ways of involving students in their own education, from college and beyond, by developing personal learning environments, formal and informally.

\subsection{Approaches}

We examine some of the constraints for students' learning in HE, primarily from a United Kingdom perspective, with implications across the international sector. We examine how the concept of 'active learning', especially by way of out-of-classroom or fieldwork activities, can aid individual students. This is especially important for accessibility issues and the delivery of high quality education. The ubiquity of 'smart' devices and the promotion of mobile-learning (m-learning) shows that smart phones and tablets can contribute to more formal educational scenarios [1]. We suggest that a tutor-based approach, developed via smart devices, involves and integrates students' personalized learning within established structures. 


\subsection{Institutional Higher Education}

The massification and marketisation of HE is evident in many countries [2]. In the UK, universities also need to response to governmental pressures; including monitoring of overseas students, research output (the Research Excellence Framework, REF) and teaching quality (Teaching Excellence Framework, TEF). Economies of scale provide return on investment yet the enlargement of universities and the HE system can result in problems for individual students. The massification of HE has meant that student learn in lecture rooms containing large numbers of students, particularly during their first year.

We all live in an uncertain and supercomplex world. Ronald Barnett [3] has argued the central role of the university should be to deal with 'supercomplexity': a world is one in which the very frameworks by which we orient ourselves to the world are themselves contested. Consequently, in their pursuit of quality, educational programs in HE need to adjust to opportunities provided by new technologies to meet the students' requirements of 'active education' and information literacy in today's world. So, how can this be achieved?

A UK report [4], 'Horizon Scanning, what will higher education look like in 2020?' takes an international viewpoint and focuses on the educational importance of MOOCs, where impact 'on pedagogy and university business models will be profound but an evolutionary shift rather than an avalanche of change'. Although undoubtedly significant for mass, open education, MOOCs are still unimportant for most students at institutions where they attend lectures and where lecture theatres are still being built to accommodate increased numbers.

A recent review of next generation of learning environments [5] considered institutional technology enhanced learning (TEL) associated with VLEs, including analytics and user experiences (UX). The section on 'emergent models' includes ideas including; conversational teaching, interfaces and platforms and conversation-led learning, environments and chatbots. The report considers the potential of user experience to facilitate on-line learning environments and describes how 'engagement in online communities is changing dramatically, from a generation of desktop computer users to mobile online-users engaging with new forms of collaboration and interaction'.

Our contribution to this discussion is this article, written as practitioners of fieldwork education and co-designers of active learning opportunities with students. We give first thought to students and their development as individuals and recognize the need for student to work outside the constraints of many aspects of higher education. 


\section{Delivery of syllabus and curriculum}

\subsection{Classes, Lectures}

A traditionalist view of higher education (HE) in the United Kingdom (UK) in the 21 st century is still one of massed students listening to lectures given by lecturers ('faculty' in the USA); an 'instructivist' approach. For many students, this 'learning' is aided by study in libraries and constitutes what remains an essentially 'Industrial Education' [6]. The challenge and the opportunity is to harness the power of technology to provide more tailored and individualized personal learning, whereas there is a danger at present that the varied needs of individual students are subsumed via instructivist educational processes due to the larger student numbers. The ultimate goal is the considered use of educational technologies can lead to top down educational processes where the needs of the individual are truly catered for.

In the UK, considerable attention is currently being paid to lecture capture systems as part of institutional responses to quality provision by technology-enhanced education. Recorded lectures do have advantages, especially for students with disabilities or who might be in hospital, but they are still part of an institutional, instructivist, pedagogic template. Bligh's [7] book 'What's the use of lectures?' is mentioned by Phillips [8] in a brief espousal of educational design summarized in Table 1 in which theory-in-use is basically lecture-driven.

Table 1 Learning environments comparing two theories, after Phillips [8].

\begin{tabular}{lll}
\hline & Espoused Theory & Theory-in-use \\
\hline Pedagogical philosophy & constructivist & instructivist \\
Approach to learning & deep & surface \\
Approach to teaching & student-centered & teacher-centered \\
Subject design & outcomes-based & content-based \\
\hline
\end{tabular}

Phillips argues that, 'it is an important role of the teacher, in a massified tertiary education sector, to assist students to develop ... generic, lifelong learning skills'. So the challenge to educators is to consider how this might this be done in a lecture context and to avoid the situation where 'College is a place where a professor's lecture notes go straight to the students' lecture notes, without passing through the brains of either' (a quote attributed to Hamilton Holt and to Mark Twain or Edwin Slosson). Holt promoted a 'Conference Plan' involving one-on-one interaction between tutor and student. This might be likened to the 'Oxford-Cambridge tutorial', based around discussion between two or three students and their tutor. In many HE institutions large student numbers do not allow such low staff-student ratios although technology can support educators in developing key skills and critical thinking.

E-, or online, learning can support lectures by, for example, MOOCs, Spocs (small private on-line courses), visits to Wikipedia, YouTube and iTUNES U etc. These excursions might be informally suggested by tutors or as part of 'flipped' classes or Just-in-Time teaching. Information literacy needs support and guidance; what are 
reliable sources? How can students find them and distinguish them from 'conspiracy theory' sites? Tutors should work with students and information professionals to develop meaningful tasks to extend teaching options and opportunities.

Various authors have suggested ways of using the lecture itself to promote smaller chunks of learning ('micro-learning') and to use lecture time for other activities such as problem solving $[9,10]$ as well as forms of problem-based learning by peer discussion. Such interventions can provide diverse opportunities for student interaction and, as we shall argue, the integrated use of technology and smart devices to support learning, thereby approaching Phillips' 'espoused theory'. MOOCs should indeed be 'designed as challenges,' not as online lectures and connectivist eMOOCs provide a more diverse learning scenario [11] .

\subsection{Some general attributes of quality.}

The report by Davies et al. [12] indicates that the UK's Teaching Excellence Framework (TEF) will, 'focus the attention of university leaders on the opportunities presented by technology-enhanced learning'. However, the report gives no suggestions as to what the technologies will be or how they should be used to aid students' learning. We now explore Gilly Salmon's ideas [13], via Fig. 1, to examine emergent technologies, missing from Davies et al. [12], that promote a more radical view of change using peripheral, new products, technologies, markets and missions.

Quadrants 1, 2 and 3 provide the basic elements of education as e-learning. Tablets and smart mobile devices are increasingly used by students for informal media consumption or social interaction so literature searching, communicating with tutors, submitting work [14] or interacting with a VLE as recognized by Phipps et al. [5] should encourage their educational use. Indeed, since the introduction of the iPad, a range of educational hardware and software have emerged but there remains a need to place smart devices in a pedagogic setting.

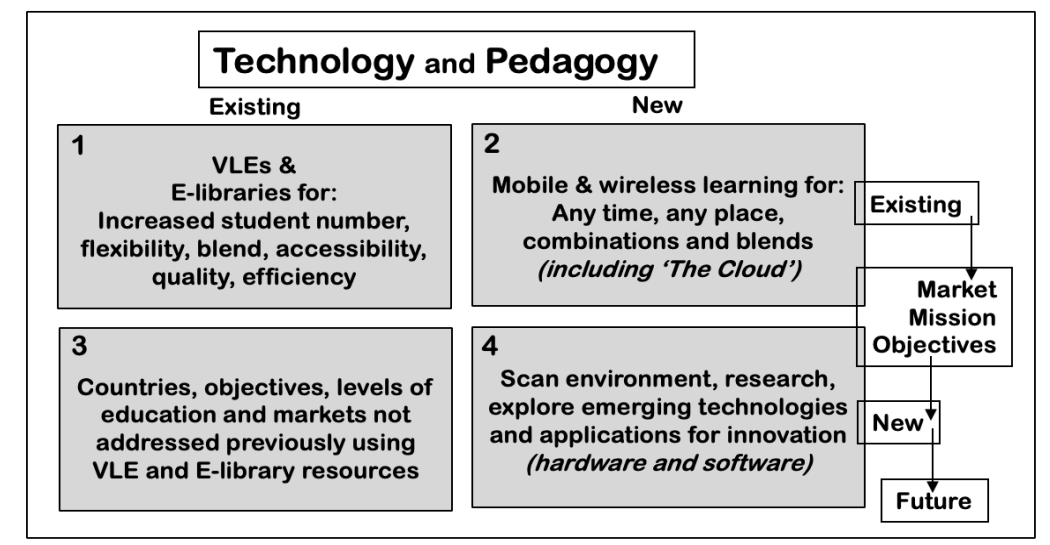

Fig. 1. New and existing practices in technology and pedagogy; modified after Salmon [13]. Additional terms in italic. 


\subsection{Education in a cognitive and inter-connected world}

There are a wide range of 'learning theories' in education, however, it is how educational principles are employed that is of importance. If technology, perhaps delivered with an institutionally-provided VLE, is used in conjunction with students' individual technology use then we could reach an optimal learning scenario as envisioned by Beetham and Sharpe [15, 16] and Laurillard [17]. If there is any basis to an overall theory, it should be based on cognitive principles [18], be adaptive to individual needs and use technological affordances wisely. Various books and publications involving metacognitive approaches are available to assist tutors design courses and employ appropriate pedagogic principles [e.g. 19]. We now examine a framework for this provision centered around a student's involvement in education.

Figure 2 uses Beetham's 'student engagement relationships', the pentangle, with types of 'learning'. Importantly, the Personal Learning Environment (PLE), is studentcentered and education is appreciative of cognition, especially meta-cognition. The idea of a Personal Learning Environment (PLE) is extended from an educational device linked to a VLE and thus rooted in Salmon's Figure 1. Dabbagh and Kitsantas [20] discuss how PLEs can, 'serve as platforms for both integrating formal and informal learning and fostering self-regulated learning in higher education contexts'. Their framework includes blogs, wikis and social networking leading from personal information management to social interaction and collaboration to information aggregation and management. Our view extends this use of a PLE to facilitate the relationships and methods described by Beetham in Fig. 2 via a students' personalization of smart mobile devices. This is integral to the PLE and incorporates aspects of the instructor/tutors via cognitive and metacognitive assistance.

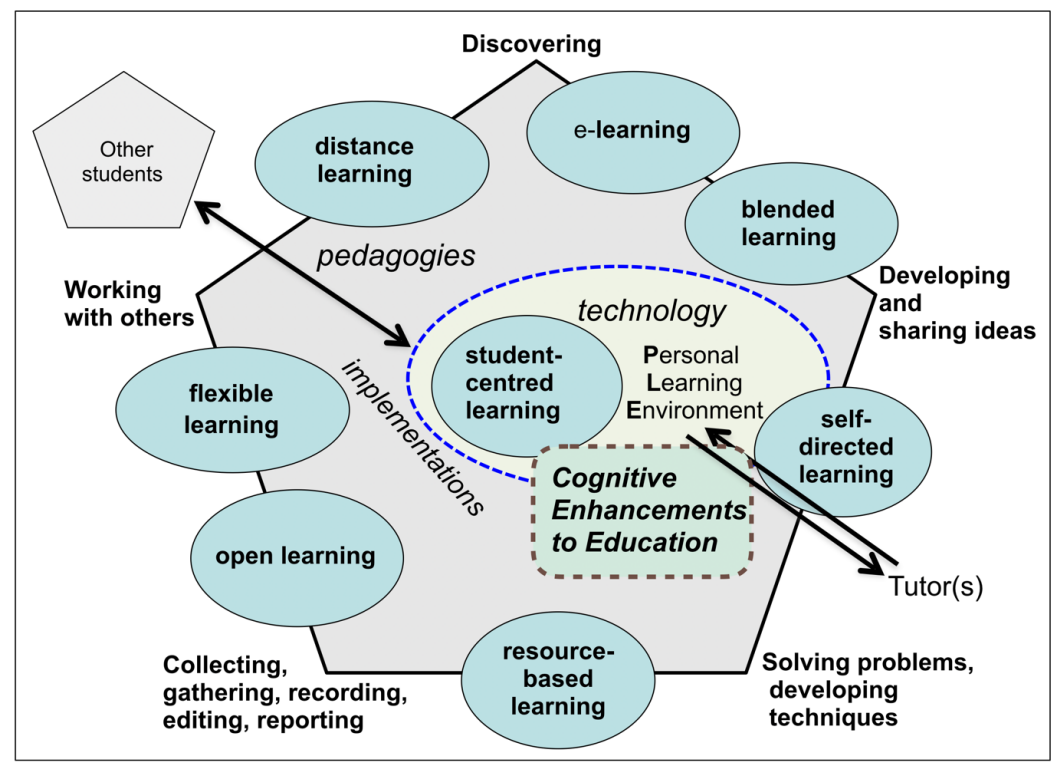

Fig. 2. Beetham's 'student engagement relationships [21] after France et al. [1]. 


\subsection{Personalization measures and the role of tutors}

Individual private tutors, such as Thomasina Coverly's tutor Septimus Hodge in the play Arcadia [22], were once commonplace for the nobility and wealthy. The modern-day equivalent can be achieved through co-developing personal learning environments (PLE) with students by tutors, lecturers, research students (graduate students) or post-doctoral researchers. They contribute to this personalization by providing one-to-few educational relationships with their students. Although these will rarely be one-to-one tutorials, a PLE relates various tutorial inputs to the individual student by placing 'student plus PLE' at the heart of individual and diverse learning experiences.

Even 'personalised' education can lead to directed learning and passive responses by students. This may often be in the form of assessments such as end of semester/year examinations rather than active, problem-solving tasks. Further, the metrics used to assess engagement in learning also may need to be re-designed. Rather than using lecture attendance to show that students are 'engaged', the USA the National Survey of Student Engagement (NSSE; nsse.indiana.edu) focuses on effective educational practices. This uses the 'seven principles for good practice in undergraduate education' [23] to promote active learning and student engagement.

These principles can certainly be used by (one-to-many) lecturers and are associated with the questions asked of students about their courses in the NSSE [24] and can be incorporated into the student engagement relationships of Figure 2 to promote active learning via a PLE. Individual, or perhaps one-to-very few, tuition can certainly promote active learning procedures [25] where tutors can respond to the learning needs of individual students, for instance providing prompt and effective feedback. Examples of the effectiveness of active learning conditions can be found in, for example, Kuh et al. [26] and Healey [27] and promoted generally by McHaney [28].

\subsection{Fieldwork and out-of-classroom learning}

Fieldwork is an important part of many academic disciplines, typically the earth, environmental and biological sciences as well as archaeology, history and some social sciences. To these may be added the more informal learning experiences when visiting holiday locations or visits to museum and art galleries. Fieldwork allows close tutor to student involvement in a way that lecturing at students does not. Fieldwork allows all of the attributes of Chickering and Gamson's seven principles to be exploited, whether or not technological devices are used. Our project, Enhancing Fieldwork Learning (EFL) has, since 2010, been promoting fieldwork and appropriate technologies designed to enhance learning opportunities for a wide range of students in fieldwork. Our experiences using smart devices, especially iPad tablets, helps promote student involvement and active learning. [29-31]. We show that a variety of 
apps can be used for many learning activities including fieldwork observation, measurement, sketching as well as note-taking and writing.

\section{Mobile technologies and personalization}

Beetham and Sharpe [32 p. 4] point out that digital technologies have, 'profoundly changed how ideas and practices are communicated, and what it means to be a knowledgeable or capable person'. Higher Education institutions still have banks of computers and students are expected to have 'computers', laptops at home or perhaps carried around with them, yet writing essays on laptops and submitting via a VLE is hardly e-learning or active learning pedagogy. In 2005 Salmon [13] indicated that, 'real development beyond projects by innovators has so far been modest'. This view might still be valid nearly 15 years later and still begs the question as to what elearning actually is and how it differs from 'learning' in an inter-connected world as suggested by our initial scenario list. Currently, it is only necessary to use a tablet or smartphone, with appropriate apps and perhaps a WiFi link, to give convenience to students. Such device ubiquity should be a way of promoting the best pedagogic practice and can be achieved by linking tutors to students. This indicates that mobile or m-learning is just a convenient subset of learning. Students and tutors can be linked via intelligent devices both formally; for course requirements and assessments, and informally; for conversation, keeping in touch and collaboration. This suggests a need to involve students, as co-producers or co-designers [33] in educational processes rather than as 'consumers' or 'investors'. We now make some suggestions within educational contexts to develop active learning with student involvement.

\subsection{Tablet technologies before and now}

Stemming from the introduction of the iPad in 2010, various books, papers and reports have promoted mobile learning in HE [e.g. 14] with a variety of student-help books. The Enhancing Fieldwork Learning project has shown the importance of mobile devices in fieldwork and out-of-classroom activities [1], bring-your own device (BYOD), and in (mobile) m-learning [30]. We have also shown the significance of the iPad as a 'vade mecum' with respect to students' information gathering, storage and retrieval [34]. The student on the front cover of Macdonald and Creanor's [35] 'Learning with Online and Mobile Technologies' has books, a pencil and notepad but the 'laptop' could now be replaced with a tablet and be located anywhere.

\subsection{Cognitive learning and technology}

Various models of using technology in education have been suggested. Although individual apps and procedures may exist in one or other of these classifications, the development of an educational operating system will itself be an adaptive, or an emergent, process according to the situation of the learner, as for example in Figure 2. A better way of envisioning e-learning is as an ecosystem of higher education where 
niches are filled with appropriate pedagogy-technologies supported by apps in the schema of Figure 2 and delivered by a PLE. We now elaborate on the use of apps to enable the Personal Learning Environment to be tailored for individual students' use.

\subsection{Cognition, recognition and learning via apps}

Whilst there is a move towards using human cognition in learning [18] this may still tend to be a top-down, how students 'should' be taught, approach. If personalization is to mean anything then it is evident that students should use all the tools available to them, in particular, via smart devices. Our brains are individual, by definition, thus the iPhone (used as a generic term) and ancillary devices will be more important than the VLE, especially if it can be trained or act as a tutor. This follows from Dweck's work on 'mindsets' [36]. The cognitive facilities provided by iPhones extend well beyond scanning text and translating to audio and providing text from audio speech recognition. Even simple apps can be used, once students recognize their importance and utility, to supplement their meta-cognition. Simple examples include making and prioritizing lists in problem solving, using tweets for group communication and feedback, as suggested by Dabbagh and Kitsantas [20].On a more complex level, graphic organizer tools help concept mapping [37] and visual thinking in general [38]. Image and pattern recognition techniques are increasingly common in apps, not only for recognizing faces but also in photographic collections, tree leaf identification (e.g. Leafsnap) and works of art (e.g. Smartify and Mereasy) and music (e.g. Shazzam or SoundHound)For those with visual impairments, LookTel provides spoken words for product packages and banknotes. There is little doubt that apps such as these, involving cognitive aspects of AI, will be further developed and when available on tablets will be useful to individual's learning opportunities.

\subsection{Tablet Technologies: looking to the future}

Neil Stephenson's futuristic novel The Diamond Age [39] places the reader in a recognizable 'Neo-Victorian' world a few years hence. The nanotech engineer, John Percival Hackworth, was asked to produce an advanced personal primer for a customer. The level of artificial intelligence (AI) supported by this device was used to construct the novel's sub-title, a 'Young Lady's Illustrated Primer'. The Primer is, 'designed to react to its owners' environment and teach them what they need to know to survive and develop' (Wikipedia: en.wikipedia.org/wiki/The Diamond Age). It is thus the ultimate PLE with its own evolving database and responses to the world.

We would not claim that the iPad is such a device, although it has some of the attributes of Stephenson's 'Primer' and could be developed further with existing and developing software technologies. However, iPads, especially in association with other iOS devices, such as iPhone and Apple Watch and linked to MacOS and 'the Cloud', can be developed as true personal devices that allow multitasking. They can aid an individual's education at any age. That is, they have attributes of a tutor. Students can already control their own learning, guided by tutors, peers and their own experiences so mobile devices can only assist in that personal development. We envisage even more personalized learning by projecting from the present capabilities of the iPad and iOS to those being developed which include; 'intelligent apps', 
enhanced hardware, the use of artificial intelligence (AI), virtual reality (VR) and augmented reality (AR) all delivered on high resolution screens.

\subsection{Establishing independent learning and 'emergence': Ada and Arthur}

Although we make no prescriptive statements about iPads and iPhones, and smartphones in general, HE can look to developing independent learning, especially with the aid of tutors. Students benefits from the tutor's knowledge, experience and wisdom, the tutor benefits from the inquisitiveness of the student (à propos Arcadia) perhaps by co-learning. The iPad system contributes nothing unless switched on (an exception is as a tray for a cup of tea and a biscuit). In the sense of the (Young Lady's) primer, an iPad might even replace a tutor. In Tom Stoppard's Arcadia's young tutee was Thomasina, the young lady heroine of Diamond Age was Nell, the name of the user of our integrated system PLE is Ada (harking back to Stoppard's character in Arcadia who was based on Ada Lovelace).

We generalize the tutor concept by suggesting that Ada asks an on-board addition to Siri that can be called by the individual student, Ada. The operating system for this personal tutor we name 'Arthur', named for Arthur Dent, user of 'The Book' in The Hitch Hiker's Guide to the Galaxy [40]; Adaptive Response To HUman Requests. Arthur should show emergent behavior by the use of AI (machine learning, etc), together with its owner, Ada. The term 'emergence' has been used in various ways and includes; new system features and evolution as well as properties of wholeness. Emergent behavior from an iPad (etc) will not just be the device alone, rather it will be able to respond intelligently to the queries of the young lady and help her make appropriate choices. Decision-making according to experience via creativity, problem solving abilities by Ada with Arthur's assistance.

The geographer William Kirk developed the idea of the 'behavioural environment', how people, individuals or groups', make decisions within the world of 'facts', the phenomenal environment. The behavioural environment is ordered (and sometimes 'disordered' in a supercomplex world) according to the social facts. Arthur, a quasiintelligent tutor would allow Ada to grow within developing experience.

iPads can act as a vade mecum for students using information content (on board and via the Cloud) as a way of personalizing learning needs [34]. We now suggest ways in which developing technologies, already on the horizon, might help in developing personal tutors. For example, Google's 'Pixel Buds', when paired with the Pixel 2 handset can carry out live language translations. A recently launched application, 'Spoke' is a form of workplace tutor. This emergent behavior (of device plus student) perhaps gives the 'Tutor test', when the answer from the personal device tutor, such as Arthur, is indistinguishable from a 'real' tutor.

\subsection{Tablets as inter-communication devices in education}

From the development of the smartphone-iPhone (from 2007), the iPad (from 2010) there is considerable degree of software commonality via the operating system (iOS) and to MacOS. The Apple Watch (2015) has its own watchOS, based on iOS, that 
enables it to act as a more than a basic communication and media (music, video) replay device. In particular, it can be used to replay conventional TV as well as recorded lectures. Asynchronously, we have podcasts/vodcasts that can be academic as much as social and used for feedback in various ways. More interactively, Skype, FaceTime and Google Duo are videotelephony or Voice-over-Internet protocol (VoIP) products that can be used for effective tutoring (peer-to-peer) via WiFi as well as 4-5G/LTE. Medical (especially general practitioner and paramedic) trials are underway to relieve pressures at surgeries and hospitals. For example, the Welsh trauma surgeon David Nott reports intensive care unit in hospitals under fire in Aleppo (Syria) being monitored 24/7 over a Skype link to a hospital in Washington DC [41] and it has long been known that machine intelligent terminals can offer simple medical advice on an impersonal basis that is acceptable for patients. Finally, various aspects of computer-mediated communication and socio-emotional content have been discussed for many years [42].

Existing developments can aid the user of a smart device in an inter-connected world. Circle of 6 (www.circleof6app.com) was originally designed for college students to prevent, or at least warn, of sexual violence. It could also be useful for students needing to foster safe relationships. This is not relying on AI in the app but rather just using the capabilities of the device. A 'group mind', often referred to as a 'hive mind', offers collective conscience approaches to sharing ideas or information that might be useful to a student class. Showing students a problem image (whether in earth, biological or medical science or history) is a good way of getting engagement by group discussion as much as individual study. This can be used as part of flipped education or Just-in-Time teaching scenarios and to develop observational and interpretative skills. It is one form of citizen science that has been shown to be effective for class-based investigations on a small scale.

Analysis of group information is a way of linking individual responses to grouped data. For example, Reddit provides a news aggregation and content analysis website with discussion. Posts and discussions on Reddit do not themselves comprise a virtual tutor but rather indicate ways in which a tutor could help students' individual analysis of a topic. A student-tutor system, such as Ada-Arthur, might ask the user to be aware of problems of group behavior and perhaps analyze results much in the same way as a phishing scam mail or 'false news' posting might be analyzed. The tutor could offer advice on a simple question-response basis by going through a decision tree asking such questions as, what is the sender's address? is it from the company? are there spelling mistakes? for a suspected phishing e-mail? This provides a simple way of slowing down the quick, but perhaps incorrect, response to an invitation [43] that can be learned by the student from an on-board tutor. In other words, the device+tutor+hive behavior would minimize risks associated with a 'quick-click' response to e-mails. Similarly, Blue's 'practical tips for staying safe on-line' [44] could be made into a personal tutorial and be more user friendly than parent-installed safety devices. Kahneman and Tversky [43] have taught us to 'think twice' before responding to a problem. This is important when dealing with social media, regretting having sent some comment or perhaps rephrasing something, or mailing the wrong person. Some social gaffes may have far-reaching consequences. Machine learning 
algorithms could be used in various ways to recognize inappropriate responses and provide a learning experience for the student. Apple's predictive text feature, QuickType, in iOS that has a machine learning component that allows the software to build custom dictionaries. Parsing text from these dictionaries could flag a warning to the student similar to grammar and spelling checkers and translation apps operate.

\subsection{Virtual and Augmented Reality, Gamification and Fieldwork}

Cloud computing (and cloud storage) may be necessary for certain tasks; for example heavy computational use in an educational context is the provision of games. Gamification, is a current topic in learning technology [45] that may be device specific or shared via the internet, in particular related to augmented and virtual reality. The release of ARKit within iOS suggests an Apple VR headset; Virtual Reality (VR) will help in 'gamification' but could also be incorporated into pedagogic practice such as tutorials, fieldwork and laboratory techniques and data visualization. However, it does require additional hardware. Augmented Reality (AR) apps are where educators can take advantage to provide better student experiences. In our own area, fieldwork is likely to be a major recipient of such benefits. Visualization methods from the oil industry and military are likely to have important spin-offs in AR. People with disabilities should be significant benefactors from these technologies. We next examine how all these facilities and affordances can be made operational in an educational system.

\section{Cognitive Education}

\subsection{Cognitive learning and the individual}

All learning, or more fully, education, is cognitive. The previous discussion tends towards connectivist approaches as exemplified in network creation and an ecological learning system [46]. Learners, of whatever stage of development, sit within such networks. What is really needed is better direction regarding individual student's behavior in the system. Good guidance is given by Douglas N Adams definition of 'a learning experience' [47 p. 274]; "You know what a learning experience is? A learning experience is one of those things that says, 'You know that thing you just did'? don't do that". The individual needs to adapt within the educational ecological system. Arthur is viewed as an adaptive system into which individuals pursue their own path as they grow with, even within, the system, as in 'The Young Lady's Illustrated Primer'. To this end the decision-making needs to be informed by previous experience. Some experiences are presented in 'Algorithms to live by' [48] and can be tempered by the findings of Kahneman and Tversky [43] and Adams' definition of 'learning experience'. The ultimate aim may be the development and recognition of competencies rather than excellence alone within a cognitive apprenticeship. There are general cognitive approaches, as presented in Bransford et al. and aspects such as prediction errors, 'nudges' and rewards. However, apps designed for this learning 
environment must be inclusive [49] and accessibility should be a major feature of developing basic operating systems.

\subsection{Empowering Ada, expanding the curriculum, syllabus and context}

Arthur is essentially a personalized operating system (OS) between a device and the user (Ada). It is trained, initially as an app manager and would link to voice, screen or text input. This may well utilize some of the features of the device OS. Some of this will be part of the linkages between OS from Apple and developers should be able to use appropriate APIs. From this viewpoint it would act as a general personal tutor but offering advice and assistance and develop with the student.

Supercomplexity of the educational system, sitting within the modern world, is poorly countered by traditional lectures/tutorials/exam. The strategic view of Salmon (Fig. 1) needs to be developed, not just with one or two apps entered via smart devices, but as an integrated and developing educational ecology. This ecological approach sits within a broad range of educational experiences (Figure 2) and decision making. Kirk's behavioral environment, adapted by 'intelligent filters', would be a useful way of assisting learning processes for students. False news may be easy to identify and evaluate but misinformation on the web may be insidious in its effects. A 'trust gap' may exist between authorities and pressure groups and explain, for example, why even in some advanced economies vaccination of children is falling. Similarly, climate change denial may have to be countered. Information aggregation and analysis apps could be developed and installed in Arthur to support Ada's analysis and understanding of this complex world.

Assessment is an important part of education; for most students this means 'examinations'. Forms of assessment are varied and the multiple-choice question (MCQ) format, perhaps in the form of quizzes, is popular and simple and can be performed online via mobile devices. Several apps for student collaboration are used successfully in many institutions alongside visualization techniques and polling apps. The need is for instructors to be aware of these apps and to exploit them creatively.

So-called 'e-assessment' is being used to help mark students term papers, essays and reports, whether for summative or formative assessment. However, this is just an update of traditional ('Victorian') systems where a submitted text (often as PDF) is read by an instructor, marked up on the page and seen on a tablet. This may speed up the process but is still conforming to old fashioned practices. Applying several of the principles of Chickering and Gamson [23] would lead to an advance in assessment methods. We envisage a 'Virtorial', sitting within Arthur, to provide new ideas for 'iAssessment' that encourage student-centered and formative assessment. An enhanced version of MCQs, using metacognition, is given by confidence-based marking (CBM) for MCQs developed by Gardner-Medwin [50]. An ideal would be for an instructor to submit some data (in a general sense) for student comment or analysis. MCQs might be used for this and CBM would be helpful but it would be helpful if an app did the marking of text entries. Message parsers used for text analysis and XML/JSON 
operations would allow students to examine their own responses to the problems. Tutors would need to supply answers as part of the original question. The tutor's job of marking many individual returns would be done by Virtorial. All sorts of data, numeric, symbolic as well as text could be dealt with in this way. This is a project under development.

\subsection{Accessibility and inclusivity}

iPads have a range of on-board affordances that take them well beyond the simple media delivery communication device envisaged by Steve Jobs. The 'Accessibility' tab in settings shows range of assistants that allow the user to control text size and contrast, keyboard switching, and assistive touch to control of Siri. (Again, we should say that most of our work has been with MacOS and iOS systems than that other vendors have similar accessibility features and apps developed for them.) Many features already available may not be known to users or instructors whether or not they have special requirements. For example, VoiceOver (iOS) might be useful to help in fieldwork when hands are full with equipment ('Hey Siri, record these data'). Some control and access to cameras, accelerometers and microphone may already be incorporated in apps. Devices can also be controlled by eye gaze and tracking, using ARKit 2 as mentioned previously. As elsewhere, developers can use features in ways which have yet to be explored by educators.

An app to measure background noise (in dBA) in a room, lab or lecture theatre, may be valuable to indicate working conditions is already in watchOS. The microphone can be used to append audio notes to written material. Similarly, the iOS 'Magnifier' features could be used as a recording microscope for field or laboratory use as well as being helpful for the visually impaired. Students' instructors may be unaware of these possibilities and our project has helped to show lecturers and tutors how they can be used. Arthur could be used, through Siri, to provide reminders or prompts to students or tutors that a feature or app is available. In fieldwork for example, expensive slope measurement devices ( $\$ 100$ for a clinometer) can be replaced by an on-board app for a few cents that each student can obtain.

Care needs to be taken to be inclusive and allow for a wide range of 'impairments' - from which we all suffer to one extent or another, old and young. Base computer operating systems already provide for a range of disabilities, which is where mobile devices score well, but attention needs also to be given to cognitive disadvantages from poor short-term/working memories through cognitive degeneration to SpLDs. James and Linda Nuttall [51] produced 'Dyslexia and the iPad, Overcoming dyslexia with technology'. This gives a personal account of how James became enabled by his device and many ideas and apps are mentioned. Neurodiversity is a general term that covers a range of conditions. These may be unknown to most tutors and educators, especially if they are 'neurotypical', but it is likely that 'educating the educators' with respect to cognitive functions is an important line of research especially where smart devices may well be important in diagnosis and remediation. 
Siri, and other personal digital assistants, are complex technologies using neural network technologies. The release of Core ML (machine learning) suggests developers will soon be incorporating natural language and machine vision into apps. It remains to be seen how well these technologies will be integrated into personalized learning tutors. For older people, they may be able to help combat the decline of 'fluid intelligence' (or reasoning) as well as failing memory. Personal Learning Environments coupled to tutorial assistants such as Arthur, are likely to provide important ways for individuals to overcome or combat impairments, physical or cognitive.

\section{Discussion and Future developments}

Salmon's schema (Fig. 1) suggests that quadrants 1, 2 and 3 reflect the use of technologies within the core of HE teaching. The term e-learning is still extant in many institutions - as if this was something different from 'good' education despite many years of using the term. The LMS/VLE, and recent technologies, such as video recording of lectures, are still basically top-down structures, often with didactic tendencies. Most undergraduate education is subject based and has to deliver 'content' and certainly lecture-delivery can play a part with educational technologies helping in this delivery. However, education in general tends to be assessment-driven. For many students this means examinations and consequent stresses. Opportunities for lecturers to broaden content may be limited by the necessity to complete a syllabus and teach for the test. Curriculum development and rethinking assessment [52] are part of reviewing and widening educational structures for enhancing quality. Figure 3 provides some ideas for enhancing teaching and considered by over-views such as that by Diana Laurillard [17]. Of themselves, these structures do not consider Barnett's 'supercomplexity'. We suggest that the continually developing capabilities of smart devices, not least linked into the home and the Internet of Things (IoT), needs to be recognized in education, in particular HE. It is here where students are part of Barnett's hazards. In providing quality in $\mathrm{HE}$, institutions have a responsibility to make students fully aware of the digital world and enable them to develop digital capabilities [53]. In developing capabilities, students (and academic staff) need guidance. Such guidance is not just 'pedagogic' or indeed in the use of digital and technological learning (TEL), but in using devices and cognitive capabilities of students [54].

Our experience in fieldwork education, where we encourage active learning and investigation with problem-solving, allows students to work in collaboration and for tutors to work with these small groups. The concept of 'graduateness' [55] and developing students' capabilities [56] are important parts of out-of-class activities that can be explored on campus and in the lecture theatre as well as in remote locations. Our use and promotion of iPads and iPhones is an important part of breaking down barriers, involving students and promoting decision making. 
iPads and other smart devices will not, of themselves, make people smart in the same way as Sugata Mitra's 'hole in the wall' made them smart, as opposed to 'knowing some stuff'. But students' capabilities can be extended with assistance from tutors. This assistance needs to include digital awareness and information literacies operating in a complex, indeed supercomplex world. As yet, we do not have Young Lady's primers that can, by themselves, sense the work and its information and evolve for the student. But they can be developed to grow with the student, not only acting as repositories of information, or memory devices but as guide for the best ways to proceed with lifelong learning. AI can help in this just in the same way that the game of Go can be programmed (AlphaGo) to beat 9-dan Go Masters but can also be used inform players. We suggest that iPad virtual tutors, such as Ada-Arthur, should be able to assist students in living in the world with more effectiveness and less vulnerability and risk to hazards of learning. Rather than knowledge acquisition for its own sake, the development of intelligent iPads with users can aid our collective power to act wisely, especially in a HE environment. This would accomplish a true 'personal learning environment' (PLE): PLE = tutor + tutee + device + hive. The term 'personalized learning' has been used for many years in the past, usually as an adjunct to e-learning. PLEs have been defined variously as both concept and technology, for example in the review by Fiedler and Väljataga [57]. M-, or mobile, learning [14] has been added to e-learning. It is time to drop the e- and $\mathrm{m}$ - now that we should be able to configure portable/wearable devices and make them more adaptable in hardware as well as 'lifewear'. Micro-electro-mechanical systems (MEMS) now allow customizable devices as well as sensors such as near field communication. Haptic screens with audio output, for example, might better integrate knowledge systems with tutees to help further personalizing learning. Keyboard, pencil, oral and eyecontrolled data entry and communication may be useful to any user. These attributes can be used and promoted by the development of virtual tutors as suggested in this paper. Active learning and student-centered approaches place more onus on the connected student. Connectedness applies not only with search engines and the internet but with fellow students and tutors, where devices are nodes foe collaboration and co-creation.

Developer features such as ARKit and Core ML in Apple iOS as well as the integration of IoT into Cloud technologies and the use of blockchain concepts for security suggests many advances for the near future. Enhancing Fieldwork Learning continues as project associated with the British Ecological Society and delivers practical short courses on fieldwork teaching and the use of iPads. Our ideas for teaching and student use of mobile devices should be enhanced by these softwarehardware developments promoting personal learning environments within educational ecologies.

\section{Conclusions}

We show that utility of 'smart devices' in the world and across a range of educational practices needs to be better exploited in HE. Tablets and other mobile smart devices, 
as well as their usability, need to be placed within educationally sound pedagogic environments. We place these principles into a basic ecological structure (Fig. 2) with a personal learning environment at the center. We have also suggested ways that educational principles can be implemented by iPads (for example) to enhance quality in education within inclusive curricula and active learning. Imaginative use of integrated mobile devices (such as watch+tablet) can therefore promote good practice in education' and by empowering the uniqueness of the individual learner's PLE. The Young Lady's Illustrated Primer prototype is already here via the Ada-Arthur virtual tutor concept. Its utility can be extended by software and hardware developers working with educators to bring the quotidian to school, college and higher education.

\section{References}

1. France, D., Whalley, W.B., Mauchline, A., Powell, V., Welsh, K., Lerczak, A., Park, J., Bednarz, R.: Introduction to Tablets and Their Capabilities, Enhancing Fieldwork Learning Using Mobile Technologies. Springer Briefs in Ecology. Springer, London (2015).

2. Calderon, A.: Massification continues to transform higher education. University World News, 02 September, 237 (2012).

3. Barnett, R.: Supercomplexity and the curriculum. Studies in Higher Education, 25(3), 255-265 (2000).

4. Lawton, W., Mamun, A., Angulo, T., Axel-Berg, A., Burroes, A., Katsomitros, A., Horizon Scanning: What will higher education look like in 2020, Horizon Scanning: What will higher education look like in 2020 Leadership Foundation for Higher Education, International Unit, London? (2013).

5. Phipps, L., Allen, R., Hartland, D., Bryant, P., Hussain, A., Wood, S., Rowett, S., Scott, A.-M., Krohn, A., Next Generation [Digital] Learning Environments: Present and Future, jisc.ac.uk/rd/projects/next-generation-digital-learning, Next Generation [Digital] Learning Environments: Present and Future, jisc.ac.uk/rd/projects/nextgeneration-digital-learning, JISC, Bristol (2018).

6. Robinson, K., Aronica, L.: Creative Schools. Allen Lane/Random House, London (2015).

7. Bligh, D.A.: What's the Use of Lectures? Jossey-Bass, San Francisco (2000).

8. Phillips, R.: Challenging the primacy of lectures: The dissonance between theory and practice in university teaching. Journal of University Teaching \& Learning Practice, 2(1), 2 (2005). Available at http://ro.uow.edu.au/jutlp/vol2/iss1/2.

9. Freeman, S., Eddy, S.L., McDonough, M., Smith, M.K., Okoroafor, N., Jordt, H., Wenderoth, M.P.: Active learning increases student performance in science, engineering, and mathematics. Proceedings of the National Academy of Sciences, 111(23), 8410-8415 (2014).

10. Whalley, W.B., Lecture discussion tasks: pedagogic and technological approaches to help break down barriers in lectures. In: Kapranos, P. (ed.) Sixth International Symposium for Engineering Education University of Manchester: Sheffield. 93-100 (2016).

11. Jadin, T., Gaisch, M.: eMOOCs for Personalised Online Learning: A Diversity Perspective. Research Track, Proceedings of the European MOOC Stakeholder Summit. 2016, 69-80 (2016).

12. Davies, S., Mullan, J., Feldman, P.: Rebooting learning for the digital age: What next for technology-enhanced higher education? Higher Education Policy Institute, Report 93, Oxford (2017). 
13. Salmon, G.: Flying not flapping: a strategic framework for e-learning and pedagogical innovation in higher education institutions. ALT-J (now Research in Learning Technology), 13(3), 201-218 (2005).

14. JISC. Mobile technology and m-learning. JISC, Bristol (2015)

15. Beetham, H., Designing for learning in an uncertain future, In: Beetham, H., Sharpe, R. (eds.) Rethinking pedagogy for a digital age (2nd edition), London: Routledge Routledge: London. 258-281 (2013).

16. Beetham, H., Sharpe, R.: Rethinking Pedagogy for a Digital Age: Designing and Delivering E-learning. Routledge, London (2007).

17. Laurillard, D.: Teaching as a design science: Building pedagogical patterns for learning and technology. Routledge, Abingdon/New York (2012).

18. Bransford, J.D., Brown, A.L., Cocking, R.R.: How people learn: Brain, mind, experience, and school. National Academies Press, Washington DC (2000).

19. Kukulska-Hulme, A., Traxler, J., Design principles for mobile learning, In: Beetham, H., Sharpe, R. (eds.) Rethinking pedagogy for a digital age: Designing for 21 st century learning Routledge: London. 244-257(2013).

20. Dabbagh, N., Kitsantas, A.: Personal Learning Environments, social media, and selfregulated learning: A natural formula for connecting formal and informal learning. The Internet and Higher Education, 15(1), 3-8 (2012).

21. Beetham, H., Designing for active learning in technology-rich contexts, In: Beetham, H., Sharpe, R. (eds.) Rethinking pedagogy for a digital age Routledge: New York and London. 31-48(2013)

22. Stoppard, T.: Arcadia. Faber, London (1993).

23. Chickering, A.W., Gamson, Z.F.: Seven principles for good practice in undergraduate education. AAHE Bulletin, 39(7), 3-7 (1987).

24. NSSE: Using NSSE to assess and improve undergraduate education. Lessons from the field, Volume 1. Indiana University Centre for Postsecondary Research, Bloomington, IN (2009).

25. Livingstone, D., Lynch, K.: Group project work and student-centred active learning: Two different experiences. Studies in Higher Education, 25(3), 325-345 (2000).

26. Kuh, G.D., Kinzie, J., Schuh, J.H., Whitt, E.J.: Student success in college: Creating conditions that matter. Jossey-Bass, San Francisco (2011).

27. Healey, M., Pawson, E., Solem, M.: Active learning and student engagement: International perspectives and practices in geography in higher education. Routledge, London (2013).

28. McHaney, R.: The new digital shoreline. Stylus, Sterling, VA. (2011).

29. Welsh, K.E., Mauchline, A.L., Park, J.R., Whalley, W.B., France, D.: Enhancing fieldwork learning with technology: practitioner's perspectives. Journal of Geography in Higher Education, 37(3), 399-415 (2013).

30. Whalley, W.B., Mauchline, A.L., France, D., Park, J., Welsh, K., The iPad six years on; Progress and Problems for Enhancing Mobile Learning with Special Reference to Fieldwork Education, In: Crompton, H., Traxler, J. (eds.) Mobile Learning and Higher Education Routledge: London. 8-18 (2018).

31. France, D., Whalley, W.B., Mauchline, A.L., Powell, V., Welsh, K.E., Lerczak, A., Park, J., Bednarz, R.: Enhancing Fieldwork Learning Using Mobile Technologies. Springer Briefs in Ecology. Springer, New York, London (2015).

32. Beetham, H., Sharpe, R.: Rethinking pedagogy for a digital age: Designing for 21st century learning (2nd Edition). Routledge, New York and London (2013).

33. Bovill, C., Cook-Sather, A., Felten, P., Millard, L., Moore-Cherry, N.: Addressing potential challenges in co-creating learning and teaching: overcoming resistance, navigating institutional norms and ensuring inclusivity in student-staff partnerships. Higher Education, 71(2), 195-208 (2016). 
34. Whalley, W.B., France, D., Mauchline, A.L., Welsh, K.E., Park, J., Everyday student use of iPads: a vade mecum for students' active learning, In: Baab, B., Bansavich, J., Souleles, N., Loizides, F., Mavri, A. (eds.) Proceedings of the 2nd International Conference on the use of iPads in Higher Education (ihe2016), San Francisco Cambridge Scholars Publishing. 43-61 (2016).

35. Macdonald, J., Creanor, L.: Learning with Online and Mobile Technologies. A Student Survival Guide. Gower, Farnham (2010).

36. Dweck, C.S.: Motivational processes affecting learning. American Psychologist, 41(10), 1040-1048 (1986).

37. Novak, J.D.: Concept maps and Vee diagrams: Two metacognitive tools to facilitate meaningful learning. Instructional Science, 19(1), 29-52 (1990).

38. Bryson, J.M., Ackermann, F., Eden, C., Finn, C.B.: Visible thinking: Unlocking causal mapping for practical business results. John Wiley \& Sons, Chichester (2004).

39. Stephenson, N.: The Diamond Age. Bantam, New York (1995).

40. Adams, D.N.: The Hitchhiker's Guide to the Galaxy. Heinemann, London (1995).

41. Nott, D.: War Doctor: Surgery on the Front Line. Picador, London (2019).

42. Rice, R.E., Love, G.: Electronic emotion: Socioemotional content in a computer-mediated communication network. Communication Research, 14(1), 85-108 (1987).

43. Kahneman, D.: Thinking, fast and slow. Allen Lane, London (2011).

44. Blue, V.: The smart girl's guide to privacy, practical tips for staying safe online. No Starch Press, San Francisco (2015).

45. Wiggins, B.E.: An overview and study on the use of games, simulations, and gamification in higher education. International Journal of Game-Based Learning, 6(1), 18-29 (2016).

46. Siemens, G.: Knowing Knowledge. George Siemens, (2006).

47. Adams, D.N.: The Salmon of Doubt. Macmillan, London (1992).

48. Christian, B., Griffiths, T.: Algorithms to live by: The computer science of human decisions. Collins, London (2016).

49. Criado Perez, C.: Invisible Women: Exposing Data Bias in a World Designed for Men. Chatto and Windus, London (2019).

50. Gardner-Medwin, A.R., Confidence-based marking, In: Bryan, C., Clegg, K. (eds.) Innovative Assessment in Higher Education Routledge: Abingdon. 141-149(2006).

51. Nuttall, J., Nuttall, L.: Dyslexia and the iPad. J and L Nutall, (2013).

52. Boud, D., Falchikov, N.: Rethinking Assessment in Higher Education: Learning for the Longer Term. Routledge, London (2007).

53. JISC Developing organisational approaches to digital capability. Retrieved from http://www.jisc.ac.uk/full-guide/developing-organisational-approaches-to-digitalcapability (2017), last accessed 2019/06.20.

54. McGuire, S.Y.: Teach Students How to Learn: Strategies You Can Incorporate Into Any Course to Improve Student Metacognition, Study Skills, and Motivation. Stylus, Sterling, VA (2015).

55. Hill, J., Walkington, H., France, D.: Graduate attributes: implications for higher education practice and policy: Introduction. Journal of Geography in Higher Education, 40(2), 155-163 (2016).

56. France, D., Powell, V., Mauchline, A.L., Welsh, K., Park, J., Whalley, W.B., Rewhorn, S.: Ability of students to recognize the relationship between using mobile apps for learning during fieldwork and the development of graduate attributes. Journal of Geography in Higher Education, 40(2), 182-192 (2016).

57. Fiedler, S.H., Väljataga, T.: Personal learning environments: concept or technology? International Journal of Virtual and Personal Learning Environments, 2(4), 1-11 (2011). 